\title{
Allocation of the EU Parliament seats via integer linear programming and revised quotas
}

\author{
Paolo Serafini \\ Department of Mathematics and Computer Science, University of Udine
}

\begin{abstract}
We deal with the problem of assigning seats to the European Parliament within the special requirements imposed by the rules of the EU. Since the usual rounding techniques, like in the divisor methods, may fail to satisfy these requirements, we propose to use integer linear programming (ILP) to provide at the same time rounding and satisfaction of the requirements. Using ILP makes central the choice of quotas to which the seats should be as close as possible. We investigate how the special requirements can affect the very definition of quotas, and define projective quotas. Finally we compare the various methods by using the EU Parliament data.
\end{abstract}

\section{Introduction}

This note contains some reflections after reading the document [2], also called 'The Cambridge Compromise'. The allocation of seats to the constituencies of a state is a well studied problem (see for instance [1]) and seemingly there is little else to say. All methods are designed to satisfy the following obvious requirement:

1. a constituency, i.e., a country for the European Union, must not receive less seats than a smaller country.

However, the rules for the EU Parliament introduce three new concepts which alter the usual framework (see [3]):

2. no country can receive more seats than a stated upper bound;

3. no country can receive less seats than a stated lower bound;

4. seats must satisfy the so called 'degressive proportionality' requirement.

Degressive proportionality means that the ratio population/seats should be an increasing function of the population. In other words, in a larger country more people are needed to form a seat.

Finding a simple method of assigning seats within these rules is the subject of [2]. The proposal is to first assign to each country a number of seats equal to the lower bound, and then to assign the remaining seats via a divisor method, by possibly capping the seats whenever they exceed the upper bound.

As shown in [2] the method may fail to satisfy the degressive proportionality rule. With regard to this point the authors suggest to weaken the rule by requiring degressive proportionality only before rounding. As a matter of fact it seems difficult to reconcile rounding schemes, like for instance the ones used in divisor methods or in largest remainders methods, with degressive proportionality.

Here we take a different attitude in order to comply with these rules. As they are, they seem particularly suited to model the problem via an integer linear programming (ILP) problem. The rules become hard 
constraints that must be satisfied by the seat assignment. In addition an objective function has to be added to the problem to get proportionality (in a way still to be made more precise) as much as possible.

It may be argued that using ILP is not transparent. A specific mathematical knowledge is required in order to model the problem and to solve it. However, in our opinion, also a divisor method requires some mathematical skills and is not amenable to the layman. Nowadays, linear programming packages are largely available (even on spreadsheets) and the model, given its modest size and simple structure, can be easily replicated in most governmental offices and university departments, so that educated people can check the result without any particular effort.

This statement may look in contrast with the attitude taken in [5] where verifiability for the layman of a biproportional seat assignment is pursued, with seats assigned according to the method suggested in [4]. We think that assigning seats to countries is indeed a delicate issue, but more at a governmental level, where tools to understand an approach and check a result are somehow available. On the contrary, assigning seats to parties on the basis of the votes expressed by the citizens requires some form of 'understanding' the method at the same level of the voters themselves.

The proportionality issue can be approached by defining rational numbers, so called 'quotas', to which the integer numbers representing the seats should adhere as much as possible, for instance minimizing some form of deviation from the quotas. This way the problem of assigning the seats is split into two separate problems, namely first defining the quotas to deal with proportionality, and then solving an ILP model to deal with rounding.

In order to define the quotas we may face the problem from two different points of view. On one hand we may consider the requirements 1.- 4 . just as constraints and we try to minimize a measure of the deviation of the seats from the 'natural' quotas. On the other hand we may consider the constraints as directives which involve smoothly all countries and as a consequence the concept of quotas must be revised.

The note is organized as follows. In Section 2 the problem is defined in mathematical terms. The integer linear programming problem is presented in Section 3. The concept of quotas is revised in Section 4 and projective quotas are defined. Simpler affine quotas are defined in Section 5 together with divisor quotas derived by the method proposed in [2], and the modified quotas proposed in [1]. The results are briefly discussed in Section 6 and some conclusions follow in Section 7. Finally all results are displayed in tables at the end of the note.

\section{Problem statement}

There are given $n$ countries. Their populations are $p_{1}, \ldots, p_{n}$. We assume that the countries are sorted as $p_{1}>p_{2}>\ldots>p_{n}$ (we may freely assume that there are no countries with the same population). Let

$P:=\sum_{i} p_{i}$. The house size (total number of seats in the parliament) is $H$. The number of seats to be assigned to country $i$ is $x_{i}$. The maximum number of seats for each country is $M$ and the minimum number 
is $m$. The constraints to be satisfied by the seats are

$$
\begin{gathered}
x_{1} \geq x_{2} \geq \ldots \geq x_{n} \\
\frac{p_{1}}{x_{1}}>\frac{p_{2}}{x_{2}}>\ldots>\frac{p_{n}}{x_{n}} \\
x_{1} \leq M, \quad x_{n} \geq m \\
\sum_{i} x_{i}=H
\end{gathered}
$$

In the actual case of the EU Parliament the data are $m=6, M=96, H=751$. The population data are reported in Table 1 . The constraint (1) is the requirement 1 . The constraints (2) is the degressive proportionality requirement 4 . The requirements 2 . and 3 . are the bounds (3).

Note that constraints (1) and (2) together restrict considerably the range of possible solutions. If, for instance, we consider two variables $x_{1}$ and $x_{2}$, the feasible values are those included between the straight lines $x_{2}=x_{1}$ and $x_{2}=\left(p_{2} / p_{1}\right) x_{1}$ For $p_{2}$ close to $p_{1}$ this is a narrow cone which may not include integer points satisfying also (4). Indeed the counterexample provided in [2] by extracting five European countries with almost equal populations shows that there can be no feasible solution at all.

The lower bound constraint in (3) is present in many actual laws (e.g., in the US House of Representatives we have $m=1$ ). The upper bound in (3) may be questioned. However, this is a political decision which is in the same line as the degressive proportionality.

The seat allocation must satisfy the constraints (1)-(2)-(3)-(4) and at the same time to be as proportional as possible to the respective populations. We address this problem by splitting these requirements into two separate problems.

On one hand the proportionality requirement leads to the definition of rational numbers, called quotas, which constitute the ideal proportional seat assignment if only seats were allowed to be fractional. There are many ways to define meaningful quotas. Note that, since the seats have to be close to the quotas, it makes sense to have the quotas themselves satisfying the constraints (1)-(2)-(3)-(4), although this is not strictly necessary. Perhaps the simplest way is to define the following natural quotas

$$
q_{i}=p_{i} \frac{H}{P}
$$

However, the bounds (3) may be not satisfied by the natural quotas and the degressive proportionality constraints (2) are clearly never satisfied. Hence we need to possibly find other ways of defining the quotas for the EU Parliament.

The question we address for the quota definition is whether the seat allocation has simply to satisfy the constraints while trying to be as proportional as possible to the natural quotas, or the requirements imposed by the law, including the bounds, should have an effect which spreads over all countries, even those which should be unaffected by the constraints. This should be reflected in the quotas themselves. In Section 4 we pursue the second view. 
Then the seat allocation within the stated constraints is taken care of by finding integer numbers satisfying the constraints and as close as possible to the defined quotas. This is explained in the next section.

\section{An integer linear programming model}

A first integer linear programming problem for the seat assignment minimizing the sum of deviations from given quotas $q_{i}$ is the following:

$$
\begin{aligned}
& \min \sum_{i=1}^{n} w_{i} \\
& w_{i} \geq q_{i}-x_{i} \quad i=1, \ldots, n \\
& w_{i} \geq x_{i}-q_{i} \quad i=1, \ldots, n \\
& x_{i} \geq x_{i+1} \quad i=1, \ldots, n-1 \\
& p_{i+1} x_{i} \leq p_{i} x_{i+1} \quad i=1, \ldots, n-1 \\
& \sum_{i=1}^{n} x_{i}=H \\
& x_{1} \leq M, \quad x_{n} \geq m \\
& x_{i} \text { integer } \quad i=1, \ldots, n
\end{aligned}
$$

A drawback of (5) is that optimal solutions are not guaranteed to be unique. For any two countries which are in defect (or in surplus) of seats with deviation larger than one for at least one country, we may exchange seats without altering the objective function value. For example, we might have $q_{i}=16.3$ and $q_{j}=15.6$ for two countries $i$ and $j$. Assume also that $16 p_{i}>18 p_{j}$. Then a feasible solution with $x_{i}=17$ and $x_{j}=17$ and another feasible solution with $x_{i}=18$ and $x_{j}=16$ (everything else the same) would have the same objective function value, since $w_{i}+w_{j}$ is the same in both cases. However, the first solution is clearly preferable because the maximum deviation is smaller.

In order to assign a larger penalty to larger deviations and still have a linear model we split each deviation $w_{i}$ into smaller terms $w_{i k}$ as $w_{i}=\sum_{k=1}^{K} w_{i k}$, with $0 \leq w_{i k} \leq 1$ (we have to figure out a number $K$ of terms

large enough to allow $w_{i}$ to be split into the $w_{i k}$ 's). Then each $w_{i k}$ receives the penalty coefficient $k$. Due to these coefficients in the objective function, the deviation $w_{i}$ will be first 'filled' by the terms with smallest $k$. 
Therefore it is advisable to model the seat assignment problem as the following ILP problem.

$$
\begin{array}{ll}
\min \sum_{i=1}^{n} \sum_{k=1}^{K} k w_{i k} & \\
w_{i} \geq q_{i}-x_{i} & i=1, \ldots, n \\
w_{i} \geq x_{i}-q_{i} & i=1, \ldots, n \\
w_{i}=\sum_{k=1}^{K} w_{i k} & i=1, \ldots, n \\
x_{i} \geq x_{i+1} & i=1, \ldots, n-1 \\
p_{i+1} x_{i} \leq p_{i} x_{i+1} & i=1, \ldots, n-1 \\
\sum_{i=1}^{n} x_{i}=H & \\
0 \leq w_{i k} \leq 1 & \\
& x_{1} \leq M, \quad x_{n} \geq m \\
& x_{i} \text { integer }
\end{array}
$$

It is helpful to note that the model (6) is equivalent to (5) with the linear objective function $\sum_{i=1}^{n} w_{i}$ replaced by the nonlinear objective function.

$$
\sum_{i=1}^{n}\left(\left(\left\lfloor w_{i}\right\rfloor+1\right)\left(w_{i}-\left\lfloor w_{i}\right\rfloor\right)+\sum_{k=1}^{\left\lfloor w_{i}\right\rfloor} k\right)=\sum_{i=1}^{n} \frac{\left(\left\lfloor w_{i}\right\rfloor+1\right)\left(2 w_{i}-\left\lfloor w_{i}\right\rfloor\right)}{2}
$$

Each term in this objective function is nothing but a piecewise linear convex function looking like a parabola. The above construction of expressing in a linear way the minimization of a piecewise linear convex function is a standard practice in mathematical programming.

Let us call 'adjacent' two solutions obtained one from each other by an exchange of one seat. Then we have:

Proposition 1. There are no adjacent optimal solution of (6), if the fractional parts of the quotas are different for any two countries.

Proof: We develop the proof by using the equivalent objective function (7). Assume $w_{i}=x_{i}-q_{i}$ and $w_{j}=x_{j}-q_{j}>1$. Then increasing $x_{i}$ by one increases the corresponding term in (7) by $w_{i}+1$ and decreasing $x_{j}$ by one decreases the corresponding term by $w_{j}$. Hence the objective function remains invariant only if $w_{i}+1=w_{j}$ and therefore only if the fractional parts of the quotas are equal. Assume $w_{i}=x_{i}-q_{i}$ and $w_{j}=x_{j}-q_{j}<1$. Then a decrease of $x_{j}$ by one makes the seat value to 'cross' the quota. It is not difficult to see that the objective function cannot remain invariant in this case. Assume $w_{i}=q_{i}-x_{i}<1$ and $w_{j}=x_{j}-q_{j}<1$. In this case both seat values cross their quotas and invariance holds only if the fractional parts of the quotas are equal. The remaining cases can be dealt with in the same way. 
Since we may assume that having the same fractional quotas for two countries is an event with probability zero, we are assured that almost always (6) does not have adjacent optima. Note also that having the same fractional quota is only a necessary condition to have adjacent optima and not a sufficient one.

There might be however equivalent non-adjacent optimal solutions. As can be seen from (7) the difference between two solutions is an integer number plus a linear combination with integer coefficients of fractional parts of quotas or their complements to unity. If we assume that such a linear combination can be integral with negligible probability, then (6) has a unique solution with high probability.

For the countries which are forced either to the lower or to the upper bound, the deviation is constant. So these countries do not play a role in the minimization, even if their terms are included in the objective function.

Due to the integrality of $x_{i}$ we may expect that the inequalities $p_{i+1} x_{i} \leq p_{i} x_{i+1}$ will be always satisfied as strict inequalities so that (2) is satisfied.

The model (6) can be clearly applied with any type of quotas. The seat assignment provided by (6) by using natural quotas is reported in Table 2, column 1. In column 0 we report the result of model (6) if we drop the degressive proportionality constraint. By comparing the two columns it is interesting to note that degressive proportionality has a stronger effect on the large countries than on the small ones. Indeed, to keep the ratios $p_{i} / x_{i}$ in the proper order, larger seat changes are required for larger populations.

We observe that the model (5) can be adapted to measure the relative deviation instead of the absolute deviation, by simply replacing each $w_{i} \geq q_{i}-x_{i}$ with $w_{i} \geq 1-x_{i} / q_{i}$ and each $w_{i} \geq x_{i}-q_{i}$ with $w_{i} \geq x_{i} / q_{i}-1$. As Balinski and Young ([1] p. 129) observe, "it can be argued that staying within the quota is not really compatible with the idea of proportionality at all, since it allows a much greater variance in the per capita representation of smaller states than it does for larger states'. Since the concept of minimizing the absolute deviation is very close to the concept of staying within the quota, it makes sense to use the relative deviation. However, we do not explore this direction in this note.

\section{Projective quotas}

In this section we revise the natural definition of quotas in order to embed in the quota definition the bounding constraints and the degressive proportionality requirement. We pursue the idea that these requirements should affect in a harmonious way all countries. In other words, even if the quota of a country is cut to the upper bound $M$, we still want all quotas to keep as much as possible their population proportions. A similar requirement should hold for the lower bound $m$. Also the degressive proportionality requirement should correspond to a smooth curve.

We assume that $q_{1}>M$ and $q_{n}<m$ (as in the current EU Parliament). If, for instance, $q_{1}<M$, the question can be addressed in a simpler way (see Section 5). Therefore we want to define new quotas $\tilde{q}_{i}$ such that the following constraints are satisfied

$$
\tilde{q}_{1}>\tilde{q}_{2}>\ldots>\tilde{q}_{n}
$$




$$
\begin{gathered}
\frac{p_{1}}{\tilde{q}_{1}}>\frac{p_{2}}{\tilde{q}_{2}}>\ldots>\frac{p_{n}}{\tilde{q}_{n}} \\
\tilde{q}_{1}=M, \quad \tilde{q}_{n}=m \\
\sum_{i} \tilde{q}_{i}=H
\end{gathered}
$$

The idea is to define $\tilde{q}$ as a function of the natural quotas $q$ such that the three equality constraints (10) and (11) are satisfied and, furthermore, also the inequalities (8) and (9) are satisfied. The criterion of simplicity would call for an affine function, but obviously affine functions cannot work here since they have two degrees of freedom while there are three equality constraints to be satisfied.

Hence we look for functions which are closest to the affine functions and have at least three degrees of freedom. These are the projective transformations, which are indeed linear in an extended space. In [2] it is noted that non-linear functions of power type have been proposed in the literature but they present the disadvantage of an 'unprincipled use of a power, non-robustness with respect to certain population distributions, non-transparent, relative difficulty of computation'. We share this criticism with respect to power functions and think that projective functions constitute a better choice if non-linearity has eventually to be chosen.

Hence, having in mind a projective transformation, we want to define a function

$$
\tilde{q}(q)=\frac{\alpha q+\beta}{\gamma q+1}
$$

so that each natural quota $q_{i}$ is mapped into a projective quota $\tilde{q}_{i}=\tilde{q}\left(q_{i}\right)$ as

$$
\tilde{q}_{i}=\frac{\alpha q_{i}+\beta}{\gamma q_{i}+1}
$$

where the parameters $\alpha, \beta$ and $\gamma$ are computed so that

$$
\tilde{q}_{1}=M, \quad \tilde{q}_{n}=m, \quad \sum_{i} \tilde{q}_{i}=H
$$

The effect of (12) together with (13) is to smoothly squeeze the interval $\left[q_{n}, q_{1}\right]$ into the interval $[m, M]$ by also keeping invariant the sum of the quotas. Note that the function $\tilde{q}(q)$ must be an increasing function of $q$ in order to satisfy the constraint (8) (actually the dependence should be w.r.t the populations, but since $q_{i}$ is linearly related to $p_{i}$ the analysis can be carried out directly on $q_{i}$ which leads to notationally simpler relations) and the function

$$
r(q):=\frac{q}{\tilde{q}(q)}=q \frac{\gamma q+1}{\alpha q+\beta}
$$

must be also an increasing function of $q$ in order to satisfy the constraint (9).

As for the first condition it is immediate to see that

Proposition 2. The function $\tilde{q}(q)$ is increasing if and only if $\alpha>\beta \gamma$. 
It is also easy to see that (only a sufficient condition),

Proposition 3. The function $r(q)$ is increasing if $\alpha \gamma>0$, and $\alpha>\beta \gamma>0$.

In order to satisfy the first two constraints in (13) we impose

$$
M=\frac{\alpha q_{1}+\beta}{\gamma q_{1}+1}, \quad m=\frac{\alpha q_{n}+\beta}{\gamma q_{n}+1}
$$

from which we get

$$
\begin{aligned}
& \alpha(\gamma)=\frac{\gamma\left(M q_{1}-m q_{n}\right)+M-m}{q_{1}-q_{n}} \\
& \beta(\gamma)=\frac{-\gamma q_{1} q_{n}(M-m)+m q_{1}-M q_{n}}{q_{1}-q_{n}}
\end{aligned}
$$

The value for $\gamma$ can be easily computed by imposing the third constraint in (13) and using binary search. For the EU parliament data we get

$$
\alpha=0.91458, \quad \beta=5.44076, \quad \gamma=0.00183231,
$$

so that the conditions of Proposition 2 and 3 hold.

We may wonder whether these conditions hold in general, so that the idea of using projective quotas is robust enough to work with general data.

Proposition 4. $\alpha>\beta \gamma$ if $\gamma>-1 / q_{1}$.

Proof: Just note that

$$
\alpha(\gamma)-\beta(\gamma) \gamma=\frac{(M-m)\left(1+\gamma q_{1}\right)\left(1+\gamma q_{n}\right)}{q_{1}-q_{n}}
$$

Let us define

$$
f(\gamma, q):=\frac{\alpha(\gamma) q+\beta(\gamma)}{\gamma q+1}
$$

By definition $f\left(\gamma, q_{1}\right)=M$ and $f\left(\gamma, q_{n}\right)=m$, for any $\gamma$. It is also interesting to note that

$$
f\left(-\frac{1}{q_{1}}, q\right)=m, \quad f\left(-\frac{1}{q_{n}}, q\right)=M, \quad \text { for any } q
$$

Since

$$
\frac{\partial f(\gamma, q)}{\partial \gamma}=\frac{(M-m)\left(q_{1}-q\right)\left(q-q_{n}\right)}{\left(q_{1}-q_{n}\right)(1+\gamma q)^{2}}
$$

the function $f(\gamma, q)$ is increasing w.r.t $\gamma$ for any fixed value $q_{n}<q<q_{1}$. We also have

$$
\lim _{\gamma \rightarrow \infty} f(\gamma, q)=M-\frac{q_{n}\left(q_{1}-q\right)(M-m)}{q\left(q_{1}-q_{n}\right)}=: \bar{f}(q)
$$




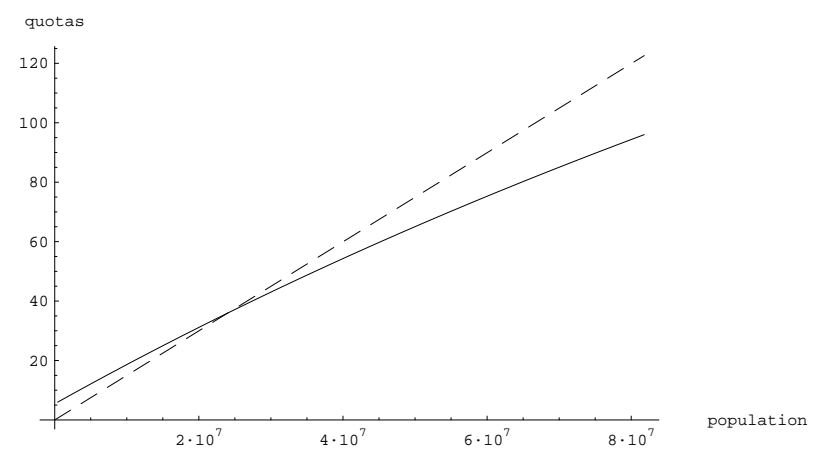

Fig. 1. Projective and natural quota functions

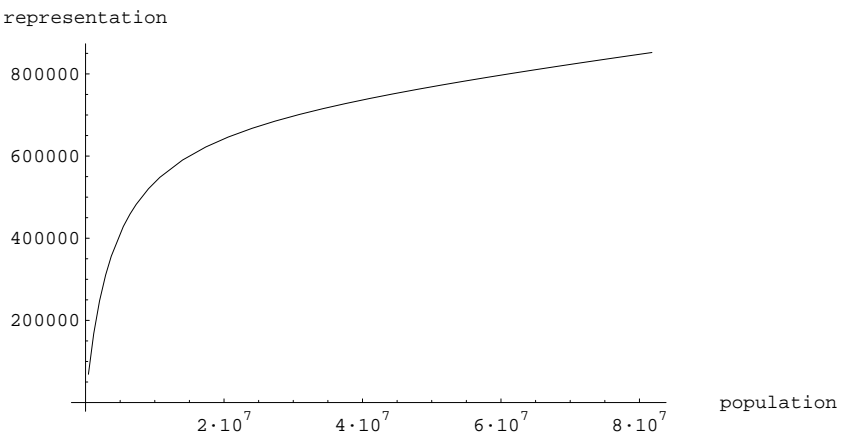

Fig. 2. Seat representation for projective quotas

Proposition 5. If $\sum_{i} \bar{f}\left(q_{i}\right)>H$, then there is a unique value $\bar{\gamma}>-1 / q_{1}$ for which $\sum_{i} f\left(\bar{\gamma}, q_{i}\right)=H$ and the function $\tilde{q}(q)$ is increasing.

Proof: From (15), one has

$$
\sum_{i} f\left(-\frac{1}{q_{1}}, q_{i}\right)=m n<H
$$

This fact, together with the hypothesis and the strictly increasing monotonicity of $f(\gamma, q)$ w.r.t. $\gamma$, proves the first statement. In turn this result together with Propositions 4 and 2 implies the second statement.

Apart from very strange instances (like for instance $n=3, q_{1}>M, q_{2}=M, q_{3}=m$, and in general with many large countries and just one very small country) one usually has

$$
\sum_{i} \bar{f}\left(q_{i}\right)>H
$$


so that we may say that in the vast majority of real cases the function $\tilde{q}(q)$ is increasing. If $\bar{\gamma}$ is positive and sufficiently small, then the conditions $\alpha \gamma>0$, and $\alpha>\beta \gamma>0$ are satisfied so that the degressive proportionality requirement is satisfied as well.

The projective quotas $\tilde{q}_{i}$ are reported in Table 1 together with the values $p_{i} / \tilde{q}_{i}$. The functions $\tilde{q}(H p / P)$ and $r(H p / P)$ are as in the Figures 1 and 2 respectively. In Figure 1 it is also shown as a dashed line the natural quota function $q(p)$.

Rounding the projective quotas may fail in general to satisfy degressive proportionality. If, for instance, we assign the seats for the EU Parliament according to the Largest Remainders rule, for which certainly (1) is satisfied, we note that (2) is not. Therefore in order to find the seat assignment we use the same ILP model (6) the only difference being that $q_{i}$ is replaced by $\tilde{q}_{i}$. The solution is reported in Table 2 , column 4 .

\section{Other quotas}

If one of the constraints (10) is missing then we may resort to simpler affine quotas, namely

$$
\hat{q}(q)=\alpha q+\beta
$$

Let us suppose that the missing constraints is the upper bound. Incidentally, this situation could arise in the future with new member countries, as pointed out in [2]. From

$$
\sum_{i}\left(\alpha q_{i}+\beta\right)=H, \quad \alpha q_{n}+\beta=m
$$

we easily get

$$
\hat{q}(q)=\frac{H-n m}{H-n q_{n}} q+\frac{H\left(m-q_{n}\right)}{H-n q_{n}}
$$

For the quotas to satisfy degressive proportionality we just need $\beta>0$ which is clearly always satisfied.

Divisor methods adjust the divisor after rounding some rational numbers which are essentially quotas. However, we may think of computing the divisor $d$ without rounding, and then we define the numbers so obtained as divisor quotas. In other words we carry out the same computation suggested in [2], i.e.

$$
A(p, d)=\min \left\{b+\frac{p}{d}, M\right\}
$$

with $b$ called the base and find the divisor $\bar{d}$ for which $\sum_{i} A\left(p_{i}, \bar{d}\right)=H$. The numbers $A\left(p_{i}, \bar{d}\right)$ are the divisor quotas. Then we round the quotas via the ILP model (6) by using the divisor quotas.

The question arises about the value to be chosen for $b$. In [2] it is suggested to take $b=m-1=5$. This choice is justified by the proposed rounding up, so that the final number of seats will always be at least $m$. However, without rounding, there is no guarantee that $A\left(p_{n}, \bar{d}\right) \geq 6$ if $b=5$. Indeed with $b=5$ we have $A\left(p_{n}, \bar{d}\right)=5.517$ 
It is therefore advisable to find that $b$ for which $A\left(p_{n}, \bar{d}\right)=6$. This can be done again by binary search in an outer loop, w.r.t. the inner loop for computing the divisor $\bar{d}$. The computation for the EU data leads to $b=5.49562$ and $\bar{d}=1.22708$. The divisor quotas computed with these values are reported in Table 1 . The seats obtained by using the divisor quotas are in Table 2, column 2 .

Divisor quotas are essentially affine quotas if the upper bound constraints are non active (or missing). In this $A(p, d)=b+p / d$ which corresponds to (16), and by computing $d$ and $b$ to satisfy the constraints we get exactly (17).

Balinski and Young ([1] pp. 133-134) propose the following modified quotas to take care of lower and upper bounds. They define the function

$$
B(q, t)= \begin{cases}m & \text { if } t q<m \\ t q & \text { if } m \leq t q \leq M \\ M & \text { if } t q>M\end{cases}
$$

with $q$ the natural quota. The modified quotas are the numbers $B\left(q_{i}, \bar{t}\right)$ with $\bar{t}$ a multiplier such that $\sum_{i} B\left(q_{i}, \bar{t}\right)=H$ (see Table 1 ). For the EU data we have $\bar{t}=1.00309$. It turns out that there is no difference in using (6) with the natural quotas or these modified quotas. Indeed trimming the natural quotas at the extremes in the very definition of the modified quotas produces the same effect as using the natural quotas and bound the seats in the ILP model (6).

Note that modified quotas and divisor quotas differ only by the way they take care of the lower bound, but this has strong impact on the quota functions and consequently on the computed seats (see Figure 3). Moreover the modified quota functions do not satisfy degressive proportionality as it is obvious from the definition, whilst the divisor quotas do, just because of the difference in the lower bound.

\section{Results}

A comparison of the seats obtained with the different methods is shown in Figure 3 and in Table 2. In Figure 3 the different quota functions are also shown.

The projective quota function is the only curve in Figure 3. The three piecewise linear functions are: the quota function (18) with the divisor $\bar{d}$ computed with rounding and $b=5$, as in [2], with breakpoint at a population of roughly $74,464,000$ people; the divisor quota function (18) with the divisor $\bar{d}$ computed without rounding and $b=5.49562$, with breakpoint at 74,101,900 (these two functions almost coincide); the modified quota (19), with breakpoints at 3,991,160 and 63,858,500.

The seats computed by using the projective quotas are the bullets $(\bullet)$ lying almost exactly on the projective quota function. The stars $(*)$ refer to the seats in [2], whereas the little squares $(\bullet)$ to the seats computed by using the divisor quotas (only the squares are visible when the seats coincide). The circles (o) are the seats computed by using the natural quotas. These are also the seats computed by using the modified quotas. The plus signs (+) refer to the present seats in the EU parliament. From the figure it can be seen that the actual seat distribution does not have a regular pattern. 


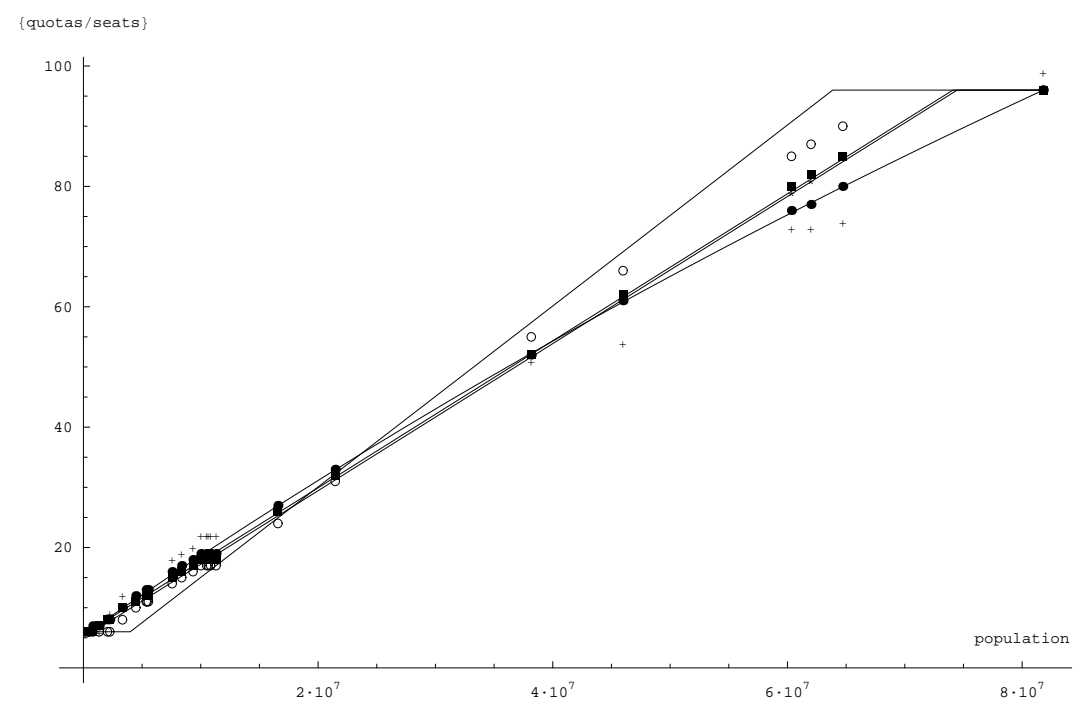

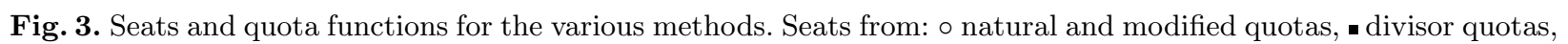
* seats in [2], • projective quotas, + actual seats

We briefly comment on Table 2. The column 0 is the allocation via ILP and natural quotas but without the constraint of degressive proportionality; the column 1 is the allocation via ILP and natural quotas (the same numbers are obtained by using ILP and modified quotas); column 2, via ILP and divisor quotas; column 3, allocation in [2]; column 4, via ILP and projective quotas. The column 5 refers to the present allocation in the EU parliament. Note that the seat sum in this column is 754 and the three extra seats are assigned to Germany. Clearly the seats in columns 1, 2 and 4 are computed with the constraint of degressive proportionality.

The seats of column 0 would be the ones most preferred by the largest countries of the EU. However, these seats do not comply with the degressive proportionality requirement. The seats of column 1 do comply with all constraints and would be the most preferred feasible seats by the largest countries. Clearly the need of being as close as possible to the natural quotas while making the smallest effort to comply with the other requirements produces seats reflecting the respective population sizes better than other methods.

The seats in columns 2 and 3 differ only in four countries. This is due to the fact that they refer to the same divisor method but with different rounding schemes. It can be checked that the difference in the four countries is just due to the degressive proportionality requirement, which is satisfied by using the ILP model.

The seats of column 4 are the ones obtained by the projective quotas. This would be the most preferred method by the smallest countries. These figures show that complying with the EU requirements to a full extent has the effect of favoring the smallest countries. 
The seats of column 5, i.e., the present situation, are by far the most penalizing for the large countries. Going from column 1 to column 5 the large countries (from Germany to Poland, comprising 70.45\% of the total population) loose seats in favor of the small countries.

\section{Conclusions}

In this note we have proposed some different methods of computing the seats in the EU Parliament. All of them do satisfy the requirements 1. - 4. exposed in the Introduction. The novelty of these methods is that they are based on integer linear programming models. This is not customary in problems of this type, for which rounding has been always taken care of in other ways. However, the usual rounding methods may fail to satisfy the degressive proportionality requirement, and it is seems plausible to say that only ILP techniques can deal efficiently with this constraint. As already outlined in the Introduction we think that ILP models are, more or less, as transparent as other methods, and therefore they can be considered as possible ways of computing seats.

The difference in seat assignments between different methods is due to the choice of quotas. The methods proposed in this note and the one proposed in [2] are all mathematically sound. Looking at the figures in Table 2 the methods can be 'ordered' according to the seats given to large countries. Clearly the question of the choice of one particular method seems at this point more a political issue than a mathematical one.

\section{References}

1. M.L. Balinski and H.P. Young: Fair representation-Meeting the ideal of one man, one vote, Second Edition. Brookings Institution Press, Washington, DC (2001).

2. G. Grimmett, with J.-F. Laslier, F. Pukelsheim, V. Ramírez González, R. Rose, W. Słomczyński, M. Zachariasen, and K. Zyczkowski: The allocation between the EU Member States of the seats in the European Parliament, European Parliament, Directorate-General for Internal Policies, Policy Department C: Citizen's Rights and Constitutional Affairs, (PE 432.760) (2011).

3. A. Lamassoure and A. Severin, Rapporteurs: European Parliament Resolution on Proposal to amend the Treaty provisions concerning the composition of the European Parliament, adopted on October 11, 2007 (INI/2007/2169) (2007).

4. B. Simeone and P. Serafini: Parametric maximum flow methods for minimax approximation of target quotas in biproportional apportionment, Networks, DOI 10.1002/net, forthcoming.

5. B. Simeone and P. Serafini: Certificates of optimality: the third way to biproportional apportionment, Social Choice and Welfare, DOI: 10.1007/s00355-010-0528-8, forthcoming. 


\begin{tabular}{|c|c|c|c|c|c|c|}
\hline country & $p_{i}$ & $q_{i}$ & $\tilde{q}_{i}$ & $p_{i} / \tilde{q}_{i}$ & $A\left(p_{i}, \bar{d}\right)$ & $B\left(q_{i}, \bar{t}\right)$ \\
\hline Germany & 81802257 & 122.596 & 96.000 & 852107. & 96.000 & 96.000 \\
\hline France & 64714074 & 96.987 & 79.937 & 809563. & 84.534 & 96.000 \\
\hline UK & 62008048 & 92.931 & 77.275 & 802431. & 81.229 & 93.218 \\
\hline Italy & 60340328 & 90.432 & 75.618 & 797964. & 79.192 & 90.711 \\
\hline Spain & 45989016 & 68.923 & 60.799 & 756417. & 61.664 & 69.136 \\
\hline Poland & 38167329 & 57.201 & 52.277 & 730104. & 52.111 & 57.378 \\
\hline Romania & 21462186 & 32.165 & 32.918 & 651983. & 31.708 & 32.265 \\
\hline Netherlands & 16574989 & 24.841 & 26.934 & 615399. & 25.739 & 24.918 \\
\hline Greece & 11305118 & 16.943 & 20.306 & 556738. & 19.303 & 16.995 \\
\hline Belgium & 10839905 & 16.246 & 19.712 & 549915. & 18.735 & 16.296 \\
\hline Portugal & 10637713 & 15.943 & 19.453 & 546833. & 18.488 & 15.992 \\
\hline Czech R. & 10506813 & 15.746 & 19.286 & 544797. & 18.328 & 15.795 \\
\hline Hungary & 10014324 & 15.008 & 18.654 & 536842. & 17.727 & 15.055 \\
\hline Sweden & 9340682 & 13.999 & 17.788 & 525125. & 16.904 & 14.042 \\
\hline Austria & 8375290 & 12.552 & 16.540 & 506362 . & 15.725 & 12.591 \\
\hline Bulgaria & 7563710 & 11.336 & 15.486 & 488407. & 14.734 & 11.371 \\
\hline Denmark & 5534738 & 8.295 & 12.832 & 431322 . & 12.255 & 8.321 \\
\hline Slovakia & 5424925 & 8.130 & 12.688 & 427579. & 12.121 & 8.155 \\
\hline Finland & 5351427 & 8.020 & 12.591 & 425027. & 12.032 & 8.045 \\
\hline Ireland & 4467854 & 6.696 & 11.425 & 391075. & 10.952 & 6.717 \\
\hline Lithuania & 3329039 & 4.989 & 9.913 & 335820 . & 9.562 & 6.000 \\
\hline Latvia & 2248374 & 3.370 & 8.470 & 265444. & 8.242 & 6.000 \\
\hline Slovenia & 2046976 & 3.068 & 8.200 & 249619. & 7.996 & 6.000 \\
\hline Estonia & 1340127 & 2.008 & 7.251 & 184821. & 7.132 & 6.000 \\
\hline Cyprus & 803147 & 1.204 & 6.527 & 123046. & 6.477 & 6.000 \\
\hline Luxembourg & 502066 & .752 & 6.120 & 82030. & 6.109 & 6.000 \\
\hline Malta & 412970 & .619 & 6.000 & 68828. & 6.000 & 6.000 \\
\hline
\end{tabular}

Table 1. Different quotas 


\begin{tabular}{|l|c|c|c|c|c|c|}
\hline country & 0 & 1 & 2 & 3 & 4 & 5 \\
\hline \hline Germany & 96 & 96 & 96 & 96 & 96 & 99 \\
\hline France & 96 & 90 & 85 & 85 & 80 & 74 \\
\hline UK & 93 & 87 & 82 & 81 & 77 & 73 \\
\hline Italy & 91 & 85 & 80 & 79 & 76 & 73 \\
\hline Spain & 69 & 66 & 62 & 62 & 61 & 54 \\
\hline Poland & 57 & 55 & 52 & 52 & 52 & 51 \\
\hline Romania & 32 & 31 & 32 & 32 & 33 & 33 \\
\hline Netherlands & 25 & 24 & 26 & 26 & 27 & 26 \\
\hline Greece & 17 & 17 & 18 & 19 & 19 & 22 \\
\hline Belgium & 16 & 17 & 18 & 19 & 19 & 22 \\
\hline Portugal & 16 & 17 & 18 & 18 & 19 & 22 \\
\hline Czech R. & 16 & 17 & 18 & 18 & 19 & 22 \\
\hline Hungary & 15 & 17 & 18 & 18 & 19 & 22 \\
\hline Sweden & 14 & 16 & 17 & 17 & 18 & 20 \\
\hline Austria & 13 & 15 & 16 & 16 & 17 & 19 \\
\hline Bulgaria & 12 & 14 & 15 & 15 & 16 & 18 \\
\hline Denmark & 8 & 11 & 12 & 12 & 13 & 13 \\
\hline Slovakia & 8 & 11 & 12 & 12 & 13 & 13 \\
\hline Finland & 8 & 11 & 12 & 12 & 13 & 13 \\
\hline Ireland & 7 & 10 & 11 & 11 & 12 & 12 \\
\hline Lithuania & 6 & 8 & 10 & 10 & 10 & 12 \\
\hline Latvia & 6 & 6 & 8 & 8 & 8 & 9 \\
\hline Slovenia & 6 & 6 & 8 & 8 & 8 & 8 \\
\hline Estonia & 6 & 6 & 7 & 7 & 7 & 6 \\
\hline Cyprus & 6 & 6 & 6 & 6 & 7 & 6 \\
\hline Luxembourg & 6 & 6 & 6 & 6 & 6 & 6 \\
\hline Malta & 6 & 6 & 6 & 6 & 6 & 6 \\
\hline
\end{tabular}

Table 2. Comparison of different seat assignments: 0: via natural quotas without degressive proportionality; 1: via natural quotas; 2: via divisor quotas; 3: seats as in [2]; 4: via projective quotas; 5: present allocation. 